\title{
Paleoproteomics Reveals the Species Sources of Ancient Hair Textiles and Supports Historicity of Wool Manufacture in the Bronze Age Along the Silk Road
}

\section{Zhongyuan Wang \\ Zhejiang Sci-Tech University \\ Hailiang Yang \\ China National Silk Museum}

Yujie Zhai

Zhejiang Sci-Tech University

Shanshan Song

Zhejiang Sci-Tech University

Lian Zhou

Zhejiang Sci-Tech University

Hui Yang

Zhejiang Sci-Tech University

Hailing Zheng

China National Silk Museum

Yang Zhou

China National Silk Museum

Zhiwen Hu

Zhejiang Sci-Tech University

Zhiqin Peng

Zhejiang Sci-Tech University

Bing Wang ( $\square$ wbing388@163.com )

Zhejiang Sci-Tech University

\section{Research Article}

Keywords: Paleoproteomics, Species identification, Ancient hair textiles, Silk Road

Posted Date: January 29th, 2021

DOI: https://doi.org/10.21203/rs.3.rs-144035/v1 
License: (c) (i) This work is licensed under a Creative Commons Attribution 4.0 International License. Read Full License 


\section{Abstract}

The Xinjiang region is a crossroad between the West and East on the Silk Road, where many exquisite hair textile relics in the Bronze Age were unearthed, therefore, it attracts the attention of scholars around the world. However, the limitation of information acquisition ability is still the main bottleneck to recognize the scientific value of ancient hair textile relics. In this study, paleoproteomics method was proposed to analyse the hair textile relics from Xiaohe and Shanpula cemetery in the Xinjiang region. To establish the databases of biomarkers and morphological features, five modern hair samples from representative animals (grey fox, raccoon dog, American mink, cape hare, and lamb) were selected and examined. Through searching the databases, the ancient hair textile samples from Xiaohe and Shanpula cemetery were all identified as wool. Ultimately, this work demonstrated the evidences supporting the existence of prosperous sheep husbandry, wool manufacture and cultural exchange along the Silk Road in the Bronze Age. The paleoproteomics, in combination with other methods, obtained more reliable and profound information from hair textile relics than ever before, and it goes without saying that they have bright prospects of being widely used in hair textile archaeology.

\section{Introduction}

The Silk Road was the most important trade channel between the West and East in Eurasia, starting from Chang'an city, the capital of ancient China, passing through Central Asia and reaching the Mediterranean and Europe. Through the Silk Road, Chinese silk fabrics were brought to various countries in the West, and foreign animal hair textiles and gemstones were also imported into China. As time went by, the Silk Road had become the general term for all channels of cultural and economic exchanges between China and the West.

As a cultural and geographical crossroad ${ }^{1}$ between the West and East, the Xinjiang region is a special location on the Silk Road, which has been influenced by both oasis-based agrarian states and nomadic powers ranging from prehistoric to modern. ${ }^{2}$ Moreover, the Xinjiang region is full of deserts, ${ }^{3}$ and its arid climate provides favourable conditions for the preservation of textile relics; thus, there are many famous cemeteries with well-preserved hair textiles that have been unearthed, which implies significant information about Eurasian cultural exchanges. One example is Xiaohe cemetery, ${ }^{4}$ which is well-known for its unique and mysterious culture dating back to $1,800 \mathrm{BC} .{ }^{5}$ Cattle hides were removed from slaughtered cattle on the spot to wrap boat-shaped coffins and densely wooden columns varying according to the sex of the tomb owner and stood on the sand dunes, which shows shocking reproduction worship. Shanpula cemetery $(217 \mathrm{BC}-283 \mathrm{AD})^{6}$ is another important cemetery on the Silk Road, attracting the attention of scholars around the world. The unearthed cultural relics from Shanpula cemetery are abundant and precious, including hair fabrics with exotic patterns, ${ }^{7,8}$ always being studied as a typical example of cultural exchange between East and West. However, the limitation of information acquisition ability is still the main bottleneck to access the scientific value of ancient hair textile relics. 
The hair textile relics were made from mammalian hairs. For animals, thick hairs play significant roles in protecting bodies, keeping in warmth and hiding in the surroundings with protective coloration, while for humans, animal hairs with excellent warmth, breathability, elasticity and beautiful appearance are also wonderful textile materials for use and transactions. The hair textile trade has a long history, dating back to the initial stage of the Common Era, where wool textiles spread throughout Rome. ${ }^{9-12}$ With the prevalence of the wool textile trade, an industry of husbandry ${ }^{13}$ was developed to meet the growing demand. Thus, studying animal hair relics is the key to understanding the history of textiles, not only to know about the development and dissemination of hair textiles ${ }^{14}$ but also to address issues regarding the technology, ${ }^{12}$ production economics, aesthetics, ${ }^{15}$ religion and cultural exchange between ethnic groups. $^{16-20}$

In addition to wool, there are various animal hairs that can be textile materials, such as the hairs of foxes, hares, ${ }^{21}$ raccoon dogs, minks, ${ }^{22}$ and coypus. ${ }^{23}$ Some hairs are also used directly with the skins, which are well known as furs. Among them are the general rabbit fur, and the more expensive fox fur, coypu fur, mink fur, etc. Due to the excellent properties of thermal insulation, and hydrophilic, elastic, and dyeable characteristics, animal hairs are popular worldwide as materials to make coats, scarves, and hats. However, a problem within archaeological textile research has emerged, where deterioration and contamination of ancient relics and the shortcoming of conventional detection methods lead to incorrect identification of the animals sourced for the hair in ancient relics.

Based on the hair structure, many methods have been developed to identify animal species, including direct observation, ${ }^{24}$ scanning electron microscopy, ${ }^{25,26}$ and fibre sectioning, ${ }^{27}$ which have been applied to study longitudinal structure ${ }^{28}$ and the cross sections of hair. ${ }^{29,30}$ Nevertheless, the features of animal hairs change with season, ${ }^{31-33}$ age and the area in which they live, and even the same kind of animal may have hairs of different colour, length, softness, thickness and scale pattern. Therefore, a qualitative and quantitative database of morphological features must be established through the analysis of a large number of distinct samples. ${ }^{34}$ Unfortunately, different animal hairs may exhibit a similar morphology, which may increase the difficulty of definite identification. In addition, Fourier transform infrared spectroscopy (FTIR), nuclear magnetic resonance (NMR), ${ }^{35} \mathrm{X}$-ray diffraction (XRD), ${ }^{26}$ amino acid analysis (AAA), and sodium dodecyl sulphate polyacrylamide gel electrophoresis (SDS-PAGE) are used to study the hair's characteristic peaks, secondary structure, ${ }^{36}$ amino acid composition, and molecular weight distribution. These methods require a relatively large number of samples, which inevitably wastes valuable hair fabric products.

In the archaeological field, ancient hair textiles composed of protein always deteriorate 37,38 by the influences of humidity, ${ }^{39}$ acidic and alkali environments, temperature, ${ }^{38}$ exposure to oxides, and microorganisms in the tomb. ${ }^{40}$ Until recently, the identification of poorly preserved hair samples remained a major challenge, which brings great difficulty to textile archaeology. As a result, an accurate and sensitive method for identifying hair textiles is in high demand. Paleoproteomics is an emerging method that has been used to study the protein composition and changes in ancient organisms. Hong et al. 
identified milk components from the furthest eastern location of prehistoric milking in the Old World to date by paleoproteomics. ${ }^{41}$ Michael et al. revealed $>4000$-year-old milk protein through paleoproteomics analysis of ancient food vessel stitching. ${ }^{42}$ Yang et al. found paleoproteomics evidence for kefir dairy in early Bronze Age China. ${ }^{43}$ Paleoproteomics is a high-throughput, definitive and sensitive tool that can be performed on low-quality samples for the detection of protein mixtures, and the above studies demonstrate the potential of paleoproteomics in archaeological applications.

In this present work, paleoproteomics and morphological methods were proposed to study the hair textile relics along the Silk Road. Above all, the databases of biomarkers and morphological features were established via analyzing five representative animal hairs (grey fox, raccoon dog, American mink, cape hare, and lamb). The species sources of hair textile relics were determined through searching the databases, and the species-diagnostic peptides were selected to verify the identification results afterwards. Then, FTIR, SDS-PAGE were developed to characterize the secondary structure and molecular weight distribution of hair samples. This research can provide profound insights into hair textile relics from appearance to structure, which possesses important significance for understanding the husbandry, textile manufacture and cultural exchange along the Silk Road in the Bronze Age. And the paleoproteomics method showed enormous potential for ultrasensitive analysis of hair textile relics, which has great application prospects for exploring the development and trade of ancient hair textiles in future study.

\section{Experimental Section}

\section{Materials and Chemicals.}

Five animal hairs and three ancient samples from Xiaohe and Shanpula cemeteries shown in Fig. 1 were kindly provided by the China National Silk Museum. The sample A, B and C are hair textile fragments falling from cloak, carpet, and skirt, respectively. All hair samples were washed twice with cleaning aqueous solution at $60^{\circ} \mathrm{C}$ for $1 \mathrm{~h}$ and then washed with flowing water to remove the grease. After drying, the samples were stored in dry and dark conditions at $20^{\circ} \mathrm{C}$.

Sodium hydroxide, $30 \%$ hydrogen peroxide, sodium dodecylbenzene sulfonate and sodium carbonate were purchased from Hangzhou Gaojing Fine Chemical Co., Ltd. Tris(hydroxymethyl) aminomethane hydrochloride, ammonium persulfate, sodium dodecyl sulfate (SDS), bromophenol blue, glycerol, glycine, $\mathrm{N}, \mathrm{N}, \mathrm{N}^{\prime}, \mathrm{N}^{\prime}$-tetramethylethylenediamine, and isopropyl alcohol were purchased from Aladdin. Dithiothreitol (DTT), urea, and ammonium bicarbonate were purchased from Sigma-Aldrich. lodoacetamide (IAA) and sequencing grade trypsin were purchased from Bio-Rad and Promega, respectively. LC/MS grade trifluoroacetic acid (TFA), formic acid (FA), and acetonitrile (ACN) were purchased from Thermo Fisher.

Solutions and buffers are as follows: ( 1 ) cleaning aqueous solution $(0.4 \%$ sodium dodecylbenzene sulfonate, $0.4 \%$ sodium carbonate); ( 2 ) aqueous extracting solution ( $2 \%$ sodium hydroxide, $0.3 \%$ hydrogen peroxide); (3) reduced loading buffer ( $0.25 \mathrm{M}$ Tris-HCl pH 6.8, $0.5 \mathrm{M} \mathrm{DTT}, 10 \%$ SDS, $0.5 \%$ 
bromophenol blue, $50 \%$ glycerol); (4) electrophoresis buffer (0.025 M Tris, $0.25 \mathrm{M}$ glycine, $0.1 \%$ SDS); (5) UA buffer (8 M urea, $0.15 \mathrm{M}$ Tris-HCl, pH 8.0); (6) lysis buffer (4\% SDS, $100 \mathrm{mM}$ Tris-HCl); (7) IAA buffer (50 mM IAA in UA); (8) trypsin buffer ( $4 \mu \mathrm{g}$ of trypsin in $40 \mu \mathrm{L}$ of $\mathrm{NH}_{4} \mathrm{HCO}_{3}$ buffer); and (9) carbonate buffer (CB) $(0.15 \%$ sodium carbonate, $0.29 \%$ sodium bicarbonate, $\mathrm{pH} 9.6)$. The water used in this study was deionized water purified with a TPM Ultrapure water system, and all reagents and chemicals were of at least of analytical grade.

\section{Sample Preparation.}

Keratin was extracted through the following steps. First, the cleaned hair was cut into 2 square millimetre pieces and dissolved in aqueous extracting solution at $60^{\circ} \mathrm{C}$ for $2 \mathrm{~h}$. Then, the insoluble portion was removed by filtration, and the filtrate was dialyzed with a dialysis membrane (3500 molecular weight cutoff) against deionized water for 3 days at room temperature. Finally, the protein solutions were lyophilized into powders for further analysis.

\section{Morphology.}

Digital images of the different animal hairs were acquired with a camera (Canon EOS700D). After being sputtered with gold at $15 \mathrm{~mA}$ for $60 \mathrm{~s}$, the hairs were observed by scanning electron microscopy (Hitachi $\mathrm{TM} 3000$ ) at $10 \mathrm{kV}$ accelerating voltage to analyse the longitudinal structures. The cross sections prepared by a Y172 fibre slicer were examined via a polarizing microscope (AxioCam MRc 5).

\section{Protein Digestion.}

One hundred milligrams of protein powder was dissolved in $600 \mu \mathrm{L}$ of lysis buffer (4\% SDS, $100 \mathrm{mM}$ Tris$\mathrm{HCl}$ ) with ultrasound in an ice bath for $1 \mathrm{~min}$, and the quantification of protein was determined by the bicinchoninic acid (BCA) method. DTT (1 M) was added to $200 \mu \mathrm{g}$ of protein solution until the final concentration of DTT reached $100 \mathrm{mM}$. After heating in a boiling water bath for $5 \mathrm{~min}$, the protein was digested by the filter-aided sample preparation (FASP) method. Then, the samples were mixed with 200 $\mu \mathrm{L}$ of UA buffer (8 M urea, $150 \mathrm{mM}$ Tris-HCl, $\mathrm{pH}$ 8.0) and centrifuged at 12,000 $\mathrm{g}$ for $15 \mathrm{~min}$ in a $10 \mathrm{kD}$ ultrafiltration centrifuge tube. Another $200 \mu \mathrm{L}$ of UA buffer was added, and the tubes were centrifuged as before with the filtrate being discarded. Subsequently, the centrifuge tube was shaken with IAA (50 mM IAA in UA) at $600 \mathrm{rpm}$ for $1 \mathrm{~min}$, kept in the dark at $25^{\circ} \mathrm{C}$ for $30 \mathrm{~min}$, and centrifuged at $12,000 \mathrm{~g}$ for 10 min. Next, the samples were washed 3 times with $100 \mu \mathrm{L}$ of UA buffer via centrifugation at $12,000 \mathrm{~g}$ for $10 \mathrm{~min}$, washed another 3 times with $100 \mu \mathrm{L}$ of ammonium bicarbonate buffer at $14,000 \mathrm{~g}$ for $10 \mathrm{~min}$, then vibrated with $40 \mu \mathrm{L}$ of trypsin buffer ( $4 \mu \mathrm{g}$ of trypsin in $40 \mu \mathrm{L}$ of $\mathrm{NH}_{4} \mathrm{HCO}_{3}$ buffer) at $600 \mathrm{rpm}$ for 1 min, then at $37^{\circ} \mathrm{C}$ for $17 \mathrm{~h}$. After that, the samples were gathered by centrifugation at $12,000 \mathrm{~g}$ for $10 \mathrm{~min}$ in new collection tubes, and the peptides after enzymatic hydrolysis were desalted using a C18 StageTip and dried under vacuum. Ultimately, the dried peptides were reconstituted in $0.1 \%$ TFA, and the peptide concentration was determined by measuring its OD280 for LC-MS/MS analysis.

\section{LC-MS/MS.}


The peptide was chromatographed on an Easy nLC 1200 nanolitre flow rate chromatography system from Thermo Scientific. The buffers used were liquid $\mathrm{A}$, which consisted of $\mathrm{H}_{2} \mathrm{O}$ with $0.1 \%$ aqueous formic acid, and liquid B, which consisted of $95 \%$ acetonitrile with $0.1 \%$ formic acid. The column was equilibrated with $100 \%$ liquid A solution. The samples were injected into a Trap column $(100 \mu \mathrm{m} \times 20 \mathrm{~mm}$, $5 \mu \mathrm{m}, \mathrm{C} 18$, Dr. Maisch $\mathrm{GmbH}$ ) and subjected to gradient separation through a chromatography column $(75 \mu \mathrm{m} \times 150 \mathrm{~mm}, 3 \mu \mathrm{m}, \mathrm{C} 18$, Dr. Maisch $\mathrm{GmbH})$ at a flow rate of $300 \mathrm{nl} / \mathrm{min}$. The liquid phase separation gradient was as follows: 0 min -3 min, linear gradient of liquid B from 2-7\%; 3 min - 48 min, linear gradient of liquid B from 7-35\%; 48 min - 53 min, linear gradient of liquid B from 35-90\%; 53 min - 60 min, liquid B was maintained at $90 \%$. After peptide separation, DDA (data-dependent acquisition) mass spectrometry was performed on a Q-Exactive Plus mass spectrometer from Thermo Scientific over $60 \mathrm{~min}$ of analysis time in positive ion mode. The parent ion scanning range was 300-1,800 m/z, the first-order mass spectrometry resolution was 70,000 at $\mathrm{m} / \mathrm{z}$ of 200 , the AGC target was $1 \mathrm{e} 6$, and the first-level Maximum IT was $50 \mathrm{~ms}$. Secondary mass spectrometry of each peptide was performed as follows: secondary mass spectra of the 20 most abundant parent ions (MS2scan) were acquired after every full scan. The secondary mass spectrometry resolution was $17,500 @ \mathrm{~m} / \mathrm{z}$ of 200 , the AGC target was 1e5, the level 2 Maximum IT was 50 ms, the MS2 activation type was HCD, the isolation window was 2.0 Th, and the normalized collision energy was 27.

\section{Protein Identification.}

The mass spectrometry database Discoverer software of MaxQuant 1.6.1.0 was used in this project along with the following databases from the UniProt Protein Database: the classifications of animals were family Canidae (dogs and allies, a total of 64,706 protein sequences), Neovison vison (Mustela vison, a total of 16,335 protein sequences), Oryctolagus cuniculus (rabbit, a total of 23,166 protein sequences), and Ovis aries (sheep, a total of 5,052 protein sequences); in addition, mammalian keratin libraries were used. The analysis parameter settings of MaxQuant search software were as described below: trypsin was used as the enzyme; a maximum of 2 missed cleavages were permitted; the precursor tolerances of the main search and first search and the MS/MS tolerance were set to $4.5 \mathrm{ppm}, 20 \mathrm{ppm}$ and 20 ppm, respectively; carbamidomethyl (C) was set as a fixed modification; oxidation (M) and acetyl (Protein N-term) were set as variable modifications; target-reverse was set as a database pattern; and both PSM FDR and protein FDR were set to 0.01 .

\section{Results}

\section{Morphology.}

As portrayed in Fig. 2a e, the animal hairs had different morphological characteristics, including thickness, length, density, etc. Except for lamb, the hairs of the other four animals had dense underhairs and fewer guard hairs. The hair of the grey fox (Fig. 2a), which was short and straight, had a complex color pattern. As shown in Fig. 2b, the raccoon dog's guard hairs were up to twice as long as the underhairs, while the underhairs were much denser than guard hairs. The hair of the American mink 
(Fig. 2c) had a shiny appearance because a large number of the short guard hairs reflect light. The hair of the cape hare (Fig. 2d) was dense, soft, and short, making it warm, while the hair of the lamb (Fig. 2e) was long and curly.

As previously described, the animal hairs were of 2 types, and in this paper, the underhairs were chosen to observe their scales because they account for most of the hair. The longitudinal features of the five animal hairs were quite different, as shown in Table S1 and Fig. $2 f \sim j$. The grey fox hair fibres, as shown in Fig. 2f, had tile-shaped scales, whereas the raccoon dog hair (Fig. 2g) and American mink hair (Fig. 2h) had thin strip scales. The most notable hair was cape hare hair (Fig. 2i), as the scales of the cape hare hair had a unique herringbone shape. The thickest hair fibre among the five animals belonged to the lamb, which was approximately $30 \mu \mathrm{m}$, while the other 4 animal hairs were between 10-19 $\mu \mathrm{m}$. The scales of the lamb hair were ring-shaped, as shown in Fig. 2j. The scales of hair from raccoon dogs and American minks were similar to each other, which may be because they are both from the order carnivora. A similar observation was made in the measurement of the longitudinal features, as shown in Table S1, where both the height of the scale and the ratio of diameter to scale height (d/I) from raccoon dogs and American minks were similar. Despite the similar scale structures, it is still not difficult to distinguish between them in the measurement of the longitudinal features. The scale density of the hairs from raccoon dogs was approximately $51.84 / \mathrm{mm}$, while that from American minks was approximately $80.33 / \mathrm{mm}$. Among the five animals, cape hare hair had the maximum scale density (up to $185 / \mathrm{mm}$ ), which may be attributed to the herringbone-shaped scales. In addition, lamb hair had a maximum $\mathrm{d} / \mathrm{I}$ ratio of 2.46. The closer the value of $\mathrm{d} / \mathrm{l}$ is to 1 , the closer the rectangular scale is to a perfect square; thus, the scales of the cape hare hair had the flattest ring shape.

The characteristics of the cross sections of the animal hairs are exhibited in Fig. $2 \mathrm{k} \sim 0$ and Table S2. All five animal hairs had medullary cavities. Except for lamb (Fig. 20), the distribution of cross-sectional morphologies from the other 4 animal hairs was uneven because they all have underhairs and guard hairs. For medullary cavities, the cross sections of cape hare hair (Fig. $2 n$ ) were so notable because it had not only single columns but also double columns, plurality of columns and no medullary cavity. The morphology of the cross sections of grey fox hair (Fig. 2k) was close to a circle, and we found the same result in Table S2. The ratio of the major axis to the minor axis $(\mathrm{a} / \mathrm{b})$ of grey fox hair was 1.35 , which is close to 1. Raccoon dog hair (Fig. 2l) had two types of cross sections: one was close to a circle, similar to the grey fox hair, and the other was oval; thus, the value of $\mathrm{a} / \mathrm{b}$ was 1.68 and higher than that of the grey fox hair. However, the grey fox still had a similar major axis, minor axis and cross-sectional area in the hair cross sections compared with raccoon dogs. Grey foxes and raccoon dogs are both Canidae, and they are carnivores along with American minks. Although the morphology of the cross sections from American mink (Fig. $2 \mathrm{~m}$ ) was not like those of the grey fox or raccoon dog, the American mink had a similar $\mathrm{a} / \mathrm{b}$ value compared with the raccoon dog. Moreover, the measurement of cross-sectional morphologies in cape hare hair was divided into two parts: some with at most one column medullary cavity and the other with multiple column medullary cavities. Despite the cape hare, the major axis, minor axis and cross-sectional area in the lamb were the maximum among the animals studied, which was consistent with the size of the animal bodies. In short, we established the morphological database of five 
species of animal hairs, and we found that this method was effective for original samples that had not been woven, dyed, or aged, and was especially suitable for species that were not similar in kinship. However, if it is a historical object that has lost morphological characteristics, this method may become difficult to perform and not be accurate enough.

\section{Molecular Structure.}

FTIR and XRD are well-established techniques that can yield information on the molecular conformation of animal hairs that cannot be detected by morphological methods. Both of these techniques are applied to analyse the differences in animal hairs at the molecular level. The FTIR results were shown in Figure S1a, five animal hairs exhibited similar spectra, which were consistent with the structural characteristics of the keratin. In order to distinguish the five animal hairs, secondary structures were analysed through FTIR spectra. As depicted in Figure S1b, different species were distinguished except grey fox and raccoon dogs, both of which are canines. The XRD patterns of all animal hairs were indistinguishable, conforming to the structural features of keratin, as illustrated in Figure $\mathrm{S} 1 \mathrm{~g}$. And the secondary structure could not be obtained by the XRD through peak fitting due to the overlap of a-helix and $\beta$-sheet diffraction bands. Consequently, species identification of animal hairs cannot be achieved by XRD method.

\section{Molecular weight.}

SDS-PAGE is a well-established and widely accepted experimental method for segregating proteins and analysing protein molecular weight. As shown in Figure S2a, there were no distinct differences in the staining patterns for keratin in each lane. Although the colour depth of each lane was diverse, this might owing to the colour of the animal hairs themselves, and it could not be the evidence identifying the five species of animals.

\section{Species Identification of Hair Textiles via Paleoproteomics.}

To further investigate the hair textiles, LC-MS/MS was performed to analyse the species source of the keratin extracted from the five animal hairs. The base peak chromatograms of the samples are shown in Fig. 3a. Canine samples of grey fox and raccoon dogs exhibited similarities in their chromatograms, implicating the similarity of the protein structure between these two samples before enzymatic hydrolysis. For the five animals (grey fox, raccoon dog, American mink, cape hare, lamb), the database of mammalian keratin was searched, as shown in Fig. 3b c, 88, 128, 64, 145, 102 proteins and 286, 206, $228,323,138$ peptides were detected, respectively. In order to get more in-depth information about species identification, the intensities of the top ten proteins of the five species are displayed in Table S3 with the exclusion of mismatched proteins from animals that cannot be used in textiles such as Gorilla gorilla gorillas and Tursiops truncatus. In grey fox hair, A0A3Q7RA57 and A0A3Q7SLK7 had high scores and their intensities were identified as components of keratin originating from Vulpes vulpes, the animal closest to the grey fox in the UniProt database. Keratin, type II cuticular Hb1 isoform X2 (A0A2Y9KMQ0; A0A2Y9KNE8) and keratin, type Il cuticular Hb6 (A0A2Y9KVB6) derived from Enhydra lutris kenyoni were detected, which may be because of the hybridization of Vulpes vulpes with the Enhydra lutris kenyoni 
genome. Interestingly, A0A3Q7RA57, A0A3Q7SLK7 and A0A2Y9KVB6 were also detected in American mink hairs. This could be attributed to the fact that both grey fox and American mink are from the order Carnivora. This phenomenon also occurred in cape hare hair. A0A2Y9KPT5 and A0A2Y9K0E9, ascribed to Enhydra lutris kenyoni and G9KSS7 and U6CX15 ascribed to Mustela putorius furo were found, whereas G1SEN0 ascribed to Oryctolagus cuniculus was detected with low scores. This was because the database of Leporidae keratins is extremely inadequate compared with other mammalian species of keratins in the UniProt database and the score depended on the database, making the score of G1SENO lower. In raccoon dog hair, F1P922 derived from Canis lupus familiaris was detected, while E3VW87 and P02441 derived from Ovis aries were detected only in lamb hair. Among them, E3VW87 with more unique peptides could be selected as a biomarker for discerning hair produced by Ovis aries from the other four animals.

To obtain biomarkers of the other four species, the four samples were cross-identified against the Canidae, Neovison vison and Leporidae databases (Table S4) for more precise keratin information. In cape hare hair, only G1SEN0 and G1SUH8 were detected, and G1SUH8 had more Razor + unique peptides; thus, G1SUH8 was selected as a biomarker. Both U6CZM3 and U6DQL8, with many unique peptides, existed only in American mink hair, meaning that both could be established as biomarkers. However, cross-reactivities were found when searching against the Canidae database, and keratins in more than one species are not suitable as biomarkers. Finally, A0A3Q7RKS7 with 5 unique peptides was used as the biomarker of grey fox hair, while Q6EIZ1 and F1PTX4 with 3 unique peptides were established as biomarkers of raccoon dog hair. Thus, we obtained the biomarkers of the five animal hairs. By searching these biomarkers in the proteomic results, the animal sources of hair textiles will be determined.

\section{Analyses of Ancient Hair Textiles.}

The ancient hair samples shown in Fig. 1 were provided by the China National Silk Museum. Sample A was unearthed from Xiaohe cemetery $(1800 \mathrm{BC})$, and samples $B$ and $C$ were unearthed from Shanpula cemetery (217 BC-283AD). The methods established above on the modern samples were performed for the identification of ancient samples, and the morphological results are displayed in Fig. 4a and Table S5. The digital photos (Fig. 1) show that the length and thickness of all the hair tended to be the same, unlike those animals with many guard hairs and underhairs. The scales of the three samples recorded by SEM (Fig. 4a) were all ring-shaped; however, the surface of the fibres was seriously corroded and some scales were fallen off, which may affect the measurements to some extent. The measurements of the fibres are illustrated in Table S5. The fibres of three samples were thicker than grey fox, American mink and cape hare, but close to lamb. The height of the scale was also close to that of the lamb but partially overlapped with that of the grey fox and cape hare. The density of scale was close to the American mink and lamb; nevertheless, observation of the density of scale was affected by corrosion on fibres, and therefore could not be accepted, and neither could the ratio of $d / I$. In conclusion, the morphological results of the three samples are closer to those of wool. Slicing requires a large amount of fibre, and XRD requires samples with a large weight; therefore, both of these methods are not suitable for the identification of ancient samples. The molecular structure and molecular weight distributions of ancient samples were analyzed 
by infrared spectra and SDS-PAGE, respectively. As observed in Figure S1c, the ancient samples were identified to be protein absolutely, however, the species sources of the ancient hair textiles could not be determined via secondary structure analysis, especially for the larger proportion of random conformation than that of the five modern samples (Figure S1d), which was due to the destruction of ordered structure after thousands of years of burial. As depicted by Figure S2b, the molecular weight distributions of sample $C$ was between $15-100 \mathrm{kDa}$, which was narrower than sample $A$ and sample $B$ of $15-250 \mathrm{kDa}$. This indicated the more severe aging of sample $\mathrm{C}$, consistent with the analysis results of the secondary structure.

Unlike inferring from the structure, LC-MS/MS was introduced to determine the species of samples based on the peptides present. The three ancient samples showed similarity in their base peak chromatograms, as displayed in Fig. 4b, indicating that the proteins were similar, and the three samples might be derived from the same species. The database of mammalian keratin was searched for three ancient samples, as shown in Fig. 4c $\sim$ d, 65, 89, 100 proteins and 263, 298, 319 peptides were detected, respectively. The number of proteins and peptides in ancient samples was comparable to that in modern samples, while conventional methods were influenced by the impurities and degradation of ancient samples, indicating that the paleoproteomics method is more stable and more suitable for ancient samples. The proteomic results of three ancient samples are shown in Table S6. All of the biomarkers were obtained before were searched against the proteomic results, and only E3VW87 of Ovis aries was found in the three ancient samples, as shown in Table 1. The identified peptides of E3VW87 in lamb hair and ancient samples are shown in Tables S13 S16. A total of 9, 8, and 8 species-diagnostic peptides of E3VW87 in lamb hair were detected in sample A, sample B and sample $C$, respectively. Therefore, the analysis results revealed that the species sources of all three ancient samples were sheep, verifying the conclusion of the morphological method.

Table 1

Proteins bearing species-diagnostic peptides identified in ancient hair textiles.

\begin{tabular}{|llllll|}
\hline Sample & Protein IDs & $\begin{array}{l}\text { Razor }+ \\
\text { unique peptides }\end{array}$ & $\begin{array}{l}\text { Unique } \\
\text { peptides }\end{array}$ & Score & Intensity \\
\hline Sample A & E3VW87 & 3 & 3 & 30.37 & 439330000 \\
\hline Sample B & E3VW87 & 2 & 2 & 20.832 & 175500000 \\
\hline Sample C & E3VW87 & 3 & 3 & 5.9541 & 93439000 \\
\hline
\end{tabular}

In order to further validate the identification results of ancient samples, the species-diagnostic peptides of sheep were selected through the following steps. First, the LC-MS/MS results of lamb and other four modern animal samples were searched against the corresponding species Uniprot databases, and then the unique peptides in different species were analysed by Venn diagram, as shown in Fig. 5a, the lamb possessed 93 unique peptides. Among the 93 unique peptides, the peptides that may exist in the other 4 species were screened out by blasting analysis, while the peptides that only exist in wool keratin were retained. Finally, a total of 7 reliable sheep species-diagnostic peptides were selected for the ancient 
samples verification, as displayed in Table S7, three peptides marked with an asterisk exist in biomarker E3VW87. The final identification results were shown in Fig. 5b, 5, 3, and 4 sheep species-diagnostic peptides were detected in sample A, B, and C, respectively. The sheep species-diagnostic peptide AHYDDIASR existing in protein biomarker E3VW87 were detected in all three ancient samples, and the peptides YEEEIALR existing in E3VW87 were detected both in sample A and sample C, proving the correctness of the previous paleoproteomics identification results via protein biomarkers.

\section{Discussion}

To begin with, sample $A$ was detected as the same species as sample $B$ and sample $C$, which demonstrated that even if underwent a longer period of degradation (according to the age of the cemeteries) (comparing sample A to sample B), buried in different environments (comparing sample $A$ to sample B), or degraded into peptides with smaller molecular weight (comparing sample $C$ to sample $B$ ), the species information required for the methods herein was retained and can be distinguished from other varieties. Apparently, the above methods based on modern hairs can also be applied to the identification of ancient samples, and the results suggested the stability, reliability and repeatability of morphological and paleoproteomics methods.

The preservation of hair relics was also found to be deeply affected by the coffin sealing technology. When analysing ancient samples, it is not difficult to find that the corrosion of hair from sample $C$ (Fig. 4a) is more severe than that of sample A, which is also being certificated by the lower molecular weight and proportion of a-helices. The evidences show that even though undergoing a longer burial time, the hair textiles from Xiaohe cemetery was not necessarily worse than those from Shanpula cemetery in preservation state. This may be related to the burial tradition of Xiaohe region: the coffins were wrapped in cattle hides removed from slaughtered cattle on the spot, sealing them completely without allowing entry of even a grain of sand. The almost completely sealed burial environment ensure the precious hairs be exempt from external destruction and contamination. This reveals the importance of excellent sealing technology in the preservation of textile artefacts.

Furthermore, the study on the exquisite textile from Xiaohe cemetery provided evidence supporting historicity of wool manufacture. All the three ancient samples were identified as wool, showing that wool was probably an important source of hair textiles during the period in this region. As vividly shown in Fig. 1, the fibers in the exquisite ancient textiles from Xiaohe cemetery had obvious signs of twisting and dyeing, indicating that residents of Xiaohe had mastered the mature weaving technology and dyeing process. In addition, the finding of wool textile in Xiaohe cemetery demonstrated that prosperous wool manufacture had emerged 3,800 years ago. According to previous research, ${ }^{4,44}$ the ancient Xiaohe residents were inferred to be engaged in sheep husbandry due to the discovery of abundant sheep bones, mutton remains and sheep dung. Undoubtedly, our finding provides an argument for the sheep husbandry practiced by the Xiaohe residents in the Bronze Age. 
Last but not least, the research on the ancient hair samples showed clues to economic and cultural exchanges along the Silk Road. Interestingly, E7FKQ3 derived from Capra falconeri with a score of 104.05 and an intensity of 2,198,500,000 was found in sample A, as shown in Table S6. Capra falconeri is the national animal of Pakistan and is distributed in Turkmenistan, Tajikistan, etc. But Capra falconeri was rarely found in China because China was the territory of other herbivores. The discovery of Capra falconeri protein in hair textiles from Xiaohe cemetery may be related to economic and cultural exchanges between Xinjiang and inner Asia about 3,800 years ago. In addition, Shanpula cemetery where the Sample B and Sample C were excavated is one of the most important cemetery on the southern passage of the Silk Road (114 BC- 127 AD). The cultural relics unearthed in Shanpula cemetery are abundant including a large number of living utensils, exquisite silk fabrics, bronze mirrors and hair textile with exotic patterns. Both animal hair textiles with exotic patterns and silk fabrics representing Chinese culture existed in the Shanpula cemetery simultaneously, which undoubtedly reveals the cultural diversity of Xinjiang region, and also proves the economic and cultural exchanges along the Silk Road.

\section{Conclusion}

In this research, paleoproteomics in combination with conventional methods were proposed to analyse the hair textile relics. The proposed methods revealed the species sources of ancient hair textiles unearthed from the Xiaohe and Shanpula cemetery were sheep, filling the gap of hair textile relics research in the Xinjiang region. Moreover, the regularity was found that the closer the species are in kinship, the more similar their hair morphologies and structures are, indicating the methods can be used for the identification of animal sources of other hair textiles, including the orders Carnivore, Lagomorpha, and Artiodactyla. In addition, paleoproteomics exhibited super stability, reliability and sensitivity while it only required very little sample. As a consequence, the paleoproteomics method possesses enormous potential for reliable identification of ancient hair fabrics, even traces of textiles, which has great application prospects for pursuing the development and trade history of ancient hair textiles, even for investigating the customs of ancient civilization and cultural exchanges along the Silk Road.

\section{Declarations}

\section{ASSOCIATED CONTENT}

\section{Supporting Information}

The Supporting Information is available free of charge on the ACS Publications website.

Details of experimental methods, supplementary tables, additional results and discussion (PDF)

\section{AUTHOR INFORMATION}

\section{Corresponding Author}


Zhiqin Peng - Zhejiang Sci-Tech University, Hangzhou,China; Email: pengqia06858@126.com

Bing Wang - Zhejiang Sci-Tech University, Hangzhou,China; Phone: +86-571-86843867; Email: wbing388@583163.com

\section{Author Contributions}

The manuscript was written through contributions of all authors. All authors have given approval to the final version of the manuscript. ‡These authors contributed equally.

Notes The authors declare no competing financial interest.

\section{ACKNOWLEDGMENT}

Financial support was provided by the National Key R\&D Program of China (2019YFC1520300), the National Natural Science Foundation of China (51603188; 51803237), the Ten Thousand Talent Program (W03020446; 2017R52054) and the Special Funds from the Administration of Cultural Heritage of Zhejiang Province (2020012; 2018001).

\section{References}

1. Li, C. et al. Ancient DNA analysis of desiccated wheat grains excavated from a Bronze Age cemetery in Xinjiang. Journal of Archaeological Science. 38, 115-119 https://doi.org/10.1016/j.jas.2010.08.016 (2011).

2. Xin, X. Y. \& Bian, X. Y. The Ancient Felting Techniques in Xinjiang. Adv. Mater. Res. 1048, 309-312 https://doi.org/10.4028/www.scientific (2014). .net/AMR.1048.309

3. Li, J. F. et al. Buried in Sands: Environmental Analysis at the Archaeological Site of Xiaohe Cemetery, Xinjiang, China. PLOS ONE. 8, e68957 https://doi.org/10.1371/journal.pone.0068957 (2013).

4. Yang, R. et al. Investigation of cereal remains at the Xiaohe Cemetery in Xinjiang, China. Journal of Archaeological Science. 49, 42-47 https://doi.org/10.1016/j.jas.2014.04.020 (2014).

5. Mai, H. et al. Characterization of cosmetic sticks at Xiaohe Cemetery in early Bronze Age Xinjiang, China.Scientific Reports 6,18939, doi:10.1038/srep18939 https://www.nature.com/articles/srep18939\#supplementary-information (2016).

6. Wagner, M. et al. The ornamental trousers from Sampula (Xinjiang, China): their origins and biography. Antiquity. 83, 1065-1075 https://doi.org/10.1017/S0003598X0009935X (2009).

7. Sheng, A. The Origin of Chinese Tapestry Weave: A New Hypothesis Based on Recent Studies of Archaeological Finds and Chu Material Culture In Memory of Schuyler Van Rensselaer Cammann (1912-1991). Textile History. 26, 53-74 https://doi.org/10.1179/004049695793711915 (1995).

8. Saitaer, X. \& Yang, X. M. Brief Analysis of Uyghur Folk Carpet. Adv. Mater. Res. 472-475, 466-468 https://doi.org/10.4028/www.scientific.net/AMR.472-475.466 (2012). 
9. RYDER, M. L., IRON AGE, H. A. I. R. E. D. \& ANIMAL SKINS FROM HALLSTATT, A. U. S. T. R. I. A. Oxford Journal of Archaeology. 11, 55-67 https://doi.org/10.1111/j.1468-0092.1992.tb00256.x (1992).

10. Ray, A. J. History and Archaeology of the Northern Fur Trade. Am. Antiq. 43, 26-34 https://doi.org/10.2307/279628 (1978).

11. Turgeon, L. \& French Fishers Fur Traders, and Amerindians during the Sixteenth Century: History and Archaeology. The William and Mary Quarterly. 55, 585-610 https://doi.org/10.2307/2674446 (1998).

12. Skals, I. Metal thread with animal-hair core. Studies in Conservation. 36, 240-242 https://doi.org/10.1179/sic.1991.36.4.240 (1991).

13. Brandt, L. Ã. et al. Characterising the potential of sheep wool for ancient DNA analyses. Archaeological and Anthropological Sciences. 3, 209-221 https://doi.org/10.1007/s12520-0110055-2 (2011).

14. FREl, K. M. et al. Archaeometry. 51, 252-276 https://doi.org/10.1111/j.1475-4754.2008.00396.x (2009).

15. Degano, I., Biesaga, M., Colombini, M. P. \& Trojanowicz, M. Historical and archaeological textiles: An insight on degradation products of wool and silk yarns. Journal of Chromatography A. 1218, 58375847 https://doi.org/10.1016/j.chroma.2011.06.095 (2011).

16. Good, I. \& Archaeological Textiles A Review of Current Research. Annual Review of Anthropology. 30, 209-226 https://doi.org/10.1146/annurev.anthro.30.1.209 (2001).

17. Willmott, C. From Stroud to Strouds: The Hidden History of a British Fur Trade Textile. Textile History. 36, 196-234 https://doi.org/10.1179/004049605x61564 (2005).

18. Miner, H. The Importance of Textiles in the Archaeology of the Eastern United States. Am. Antiq. 1, 181-192 https://doi.org/10.2307/275141 (1936).

19. Harris, S. \& Textiles Cloth, and Skins: The Problem of Terminology and Relationship. TEXTILE 6, 222-237, doi:10.2752/175183508X377645 (2008).

20. King, M. E. Analytical Methods and Prehistoric Textiles. Am. Antiq. 43, 89-96 https://doi.org/10.2307/279636 (1978).

21. Santos, C. G. et al. Identification of hare meat by a species-specific marker of mitochondrial origin. Meat Sci. 90, 836-841 https://doi.org/10.1016/j.meatsci.2011.10.018 (2012).

22. Prasolova, L. A. \& Trapezov, O. V. Effect of coat color genes on the hair pigmentation morphology in the american mink (Mustela vison Schr. L.). Russian Journal of Genetics. 43, 815-818 https://doi.org/10.1134/s1022795407070149 (2007).

23. Crean, J. F. Hats and the Fur Trade. Canadian Journal of Economics and Political Science. 28, 373386 https://doi.org/10.2307/139669 (1962).

24. Pilli, E. et al. Pet fur or fake fur? A forensic approach. Investigative Genetics. 5, 7 https://doi.org/10.1186/2041-2223-5-7 (2014).

25. Weisel, J. W., Nagaswami, C. \& Peterson, R. O. River otter hair structure facilitates interlocking to impede penetration of water and allow trapping of air. Canadian Journal of Zoology. 83, 649-655 
https://doi.org/10.1139/z05-047 (2005).

26. Müller, M. et al. Identification of single archaeological textile fibres from the cave of letters using synchrotron radiation microbeam diffraction and microfluorescence. Appl. Phys. A. 83, 183-188 https://doi.org/10.1007/s00339-006-3516-1 (2006).

27. Kuzuhara, A., Fujiwara, N. \& Hori, T. Analysis of internal structure changes in black human hair keratin fibers with aging using Raman spectroscopy. Biopolymers. 87, 134-140 https://doi.org/10.1002/bip.20805 (2007).

28. Hausman, L. A. The Microscopic Identification of Commercial Fur Hairs. The Scientific Monthly. 10, 70-78 (1920).

29. Hilton, H. \& Kutscha, N. P. Distinguishing Characteristics of the Hairs of Eastern Coyote, Domestic Dog, Red Fox and Bobcat in Maine. Am. Midl. Nat. 100, 223-227 https://doi.org/10.2307/2424793 (1978).

30. Körber-Grohne, U. Microscopic methods for identification of plant fibres and animal hairs from the Prince's tomb of Hochdorf, Southwest Germany. Journal of Archaeological Science. 15, 73-82 https://doi.org/10.1016/0305-4403(88)90020-9 (1988).

31. Kassenbeck, P.52-64(Springer Berlin Heidelberg).

32. Maurel, D., Coutant, C., Boissin-Agasse, L. \& Boissin, J. Seasonal moulting patterns in three fur bearing mammals: the European badger (Meles meles L.), the red fox (Vulpes vulpes L.), and the mink (Mustela vison). A morphological and histological study. Canadian Journal of Zoology. 64, 1757-1764 https://doi.org/10.1139/z86-265 (1986).

33. Russell, J. E. \& Tumlison, R. Comparison of Microstructure of White Winter Fur and Brown Summer Fur of Some Arctic Mammals. Acta Zoologica. 77, 279-282 https://doi.org/10.1111/j.14636395.1996.tb01272.x (1996).

34. Perrin, M. R. \& Campbell, B. S. Key to the mammals of the Andries Vosloo Kudu Reserve (eastern Cape), based on their hair morphology, for use in predator scat analysis. South African Journal of Wildlife Research - 24-month delayed open access. 10, 1-14 (1980).

35. Aluigi, A. et al. Study on the structure and properties of wool keratin regenerated from formic acid. International Journal of Biological Macromolecules. 41, 266-273 https://doi.org/10.1016/j.jijbiomac.2007.03.002 (2007).

36. Aluigi, A., Corbellini, A., Rombaldoni, F., Zoccola, M. \& Canetti, M. Morphological and structural investigation of wool-derived keratin nanofibres crosslinked by thermal treatment. International Journal of Biological Macromolecules. 57, 30-37 https://doi.org/10.1016/j.ijbiomac.2013.02.013 (2013).

37. Solazzo, C. et al. Modeling Deamidation in Sheep a-Keratin Peptides and Application to Archeological Wool Textiles. Anal. Chem. 86, 567-575 https://doi.org/10.1021/ac4026362 (2014).

38. Grosvenor, A. J., Morton, J. D. \& Dyer, J. M. Determination and Validation of Markers for Heat-Induced Damage in Wool Proteins. American Journal of Analytical Chemistry Vol. 03No.06, 6 https://doi.org/10.4236/ajac.2012.36056 (2012). 
39. Peacock, E. E. Biodegradation and characterization of water-degraded archaeological textiles created for conservation research. Int. Biodeterior. Biodegrad. 38, 49-59 https://doi.org/10.1016/S09648305(96)00023-6 (1996).

40. Kavkler, K. \& Demšar, A. Impact of fungi on contemporary and accelerated aged wool fibres. Polymer Degradation and Stability. 97, 786-792 https://doi.org/10.1016/j.polymdegradstab.2012.02.002 (2012).

41. Hong, C. et al. Identification of Milk Component in Ancient Food Residue by Proteomics. PLOS ONE. 7, e37053 https://doi.org/10.1371/journal.pone.0037053 (2012).

42. Buckley, M., Melton, N. D. \& Montgomery, J. Proteomics analysis of ancient food vessel stitching reveals > 4000-year-old milk protein. Rapid Communications in Mass Spectrometry. 27, 531-538 https://doi.org/10.1002/rcm.6481 (2013).

43. Yang, Y. et al. Proteomics evidence for kefir dairy in Early Bronze Age China. Journal of Archaeological Science. 45, 178-186 https://doi.org/10.1016/j.jas.2014.02.005 (2014).

44. Qiu, Z. et al. Paleo-environment and paleo-diet inferred from Early Bronze Age cow dung at Xiaohe Cemetery, Xinjiang, NW China. Quatern. Int. 349, 167-177 https://doi.org/10.1016/j.quaint.2014.03.029 (2014).

\section{Figures}
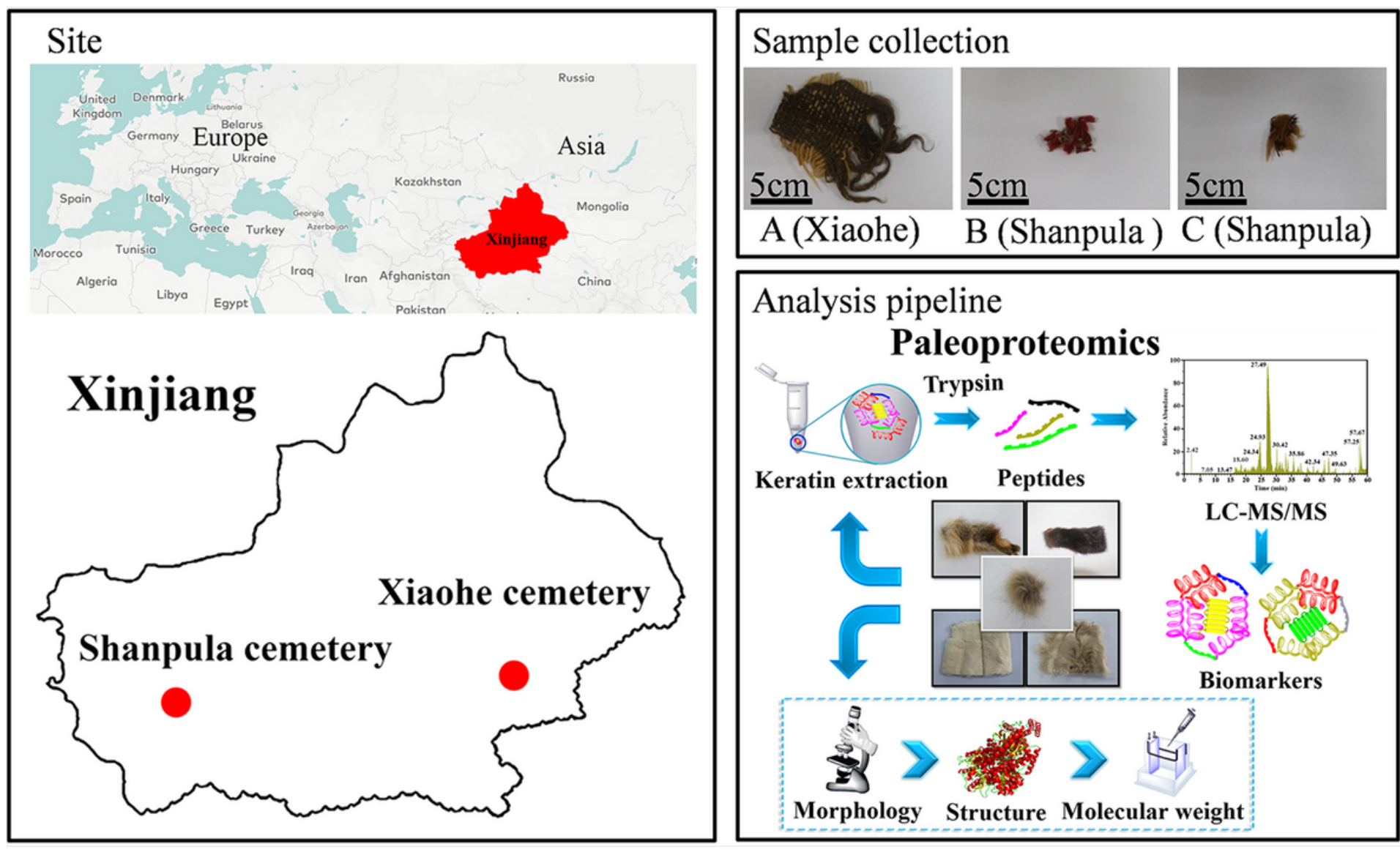

Analysis pipeline

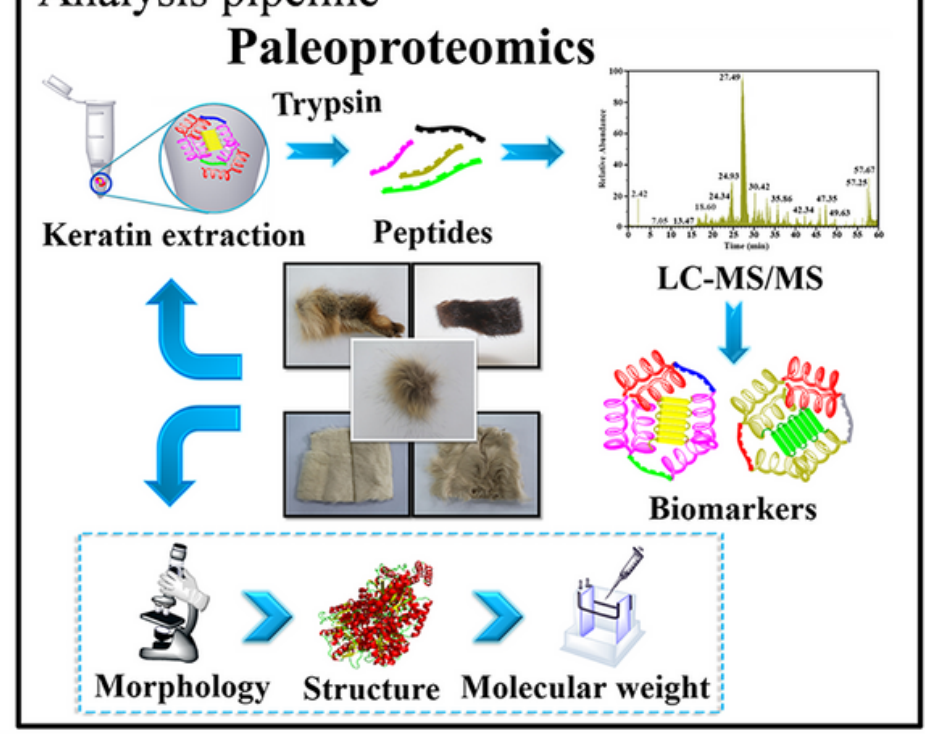

Figure 1 
Analysis workflow. Shown are the site location of ancient hair textiles, sample collection and analysis pipeline. Credits: excavation: China National Silk Museum. Note: The designations employed and the presentation of the material on this map do not imply the expression of any opinion whatsoever on the part of Research Square concerning the legal status of any country, territory, city or area or of its authorities, or concerning the delimitation of its frontiers or boundaries. This map has been provided by the authors.
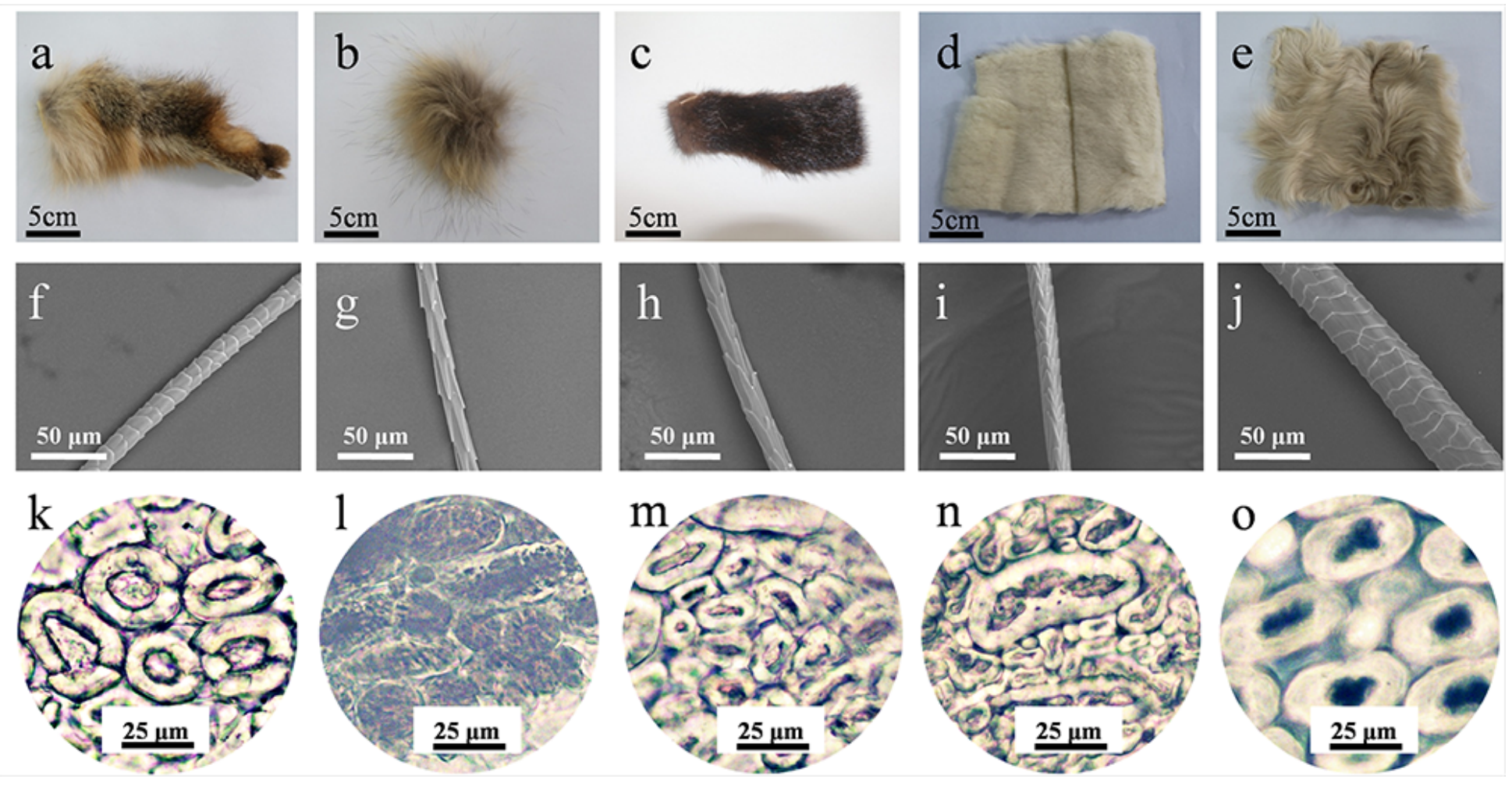

\section{Figure 2}

Morphologies of modern hairs produced by different species. Digital images of furs (top row), SEM images of the longitudinal surfaces (middle row), and photomicrographs showing cross sections of hair fibers (bottom row) for $(\mathrm{a}, \mathrm{f}, \mathrm{k})$ grey fox, $(b, g, l)$ raccoon dog, $(\mathrm{c}, \mathrm{h}, \mathrm{m})$ American mink, $(\mathrm{d}, \mathrm{i}, \mathrm{n})$ cape hare, and $(e, j, 0)$ lamb. 

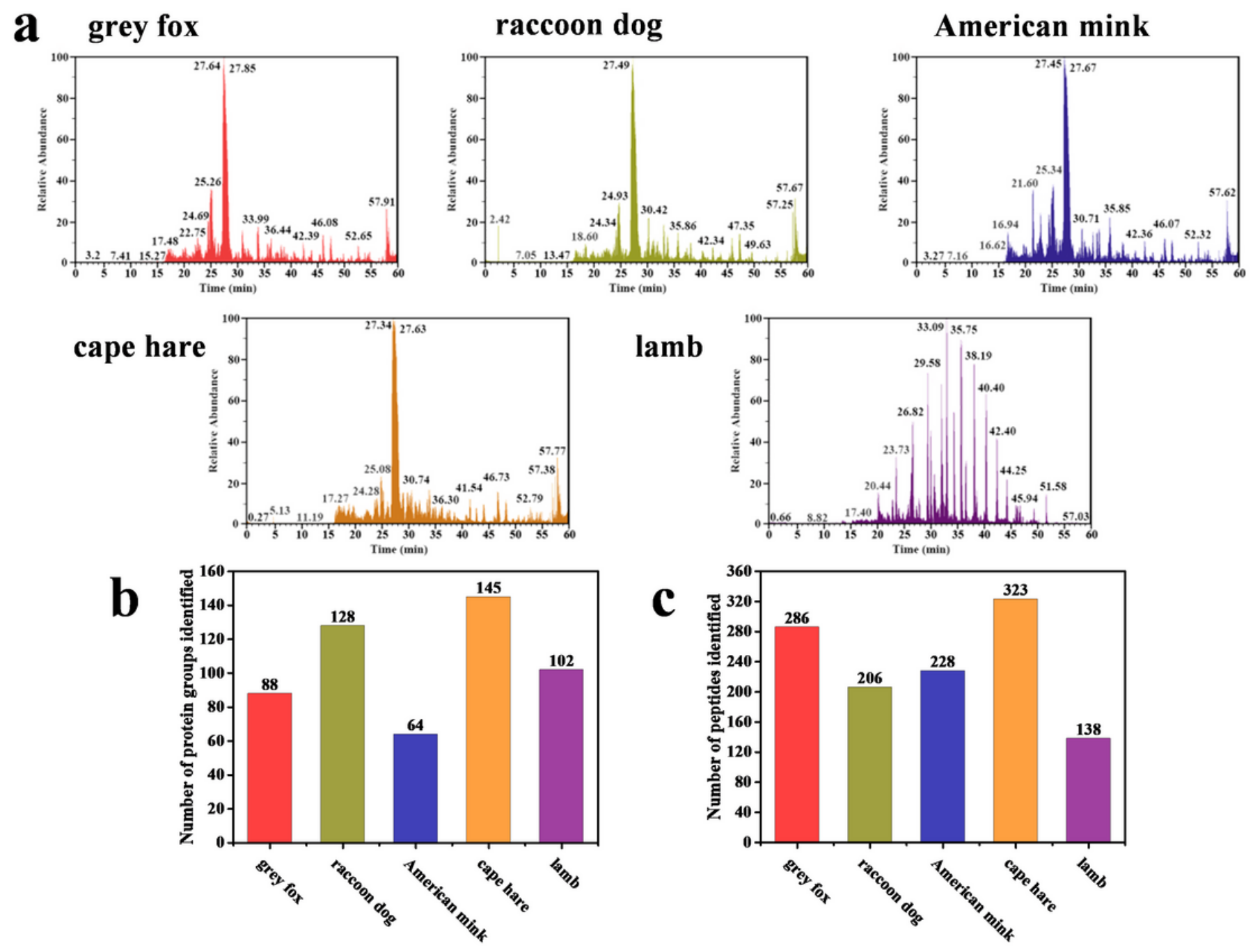

Figure 3

LC-MS/MS results of modern hair samples. (a) Base peak chromatograms of different species. (b) Protein group number and (c) peptide number of different species (grey fox, raccoon dog, American mink, cape hare, lamb) identified by comparison against the mammalian keratins Uniprot database. 

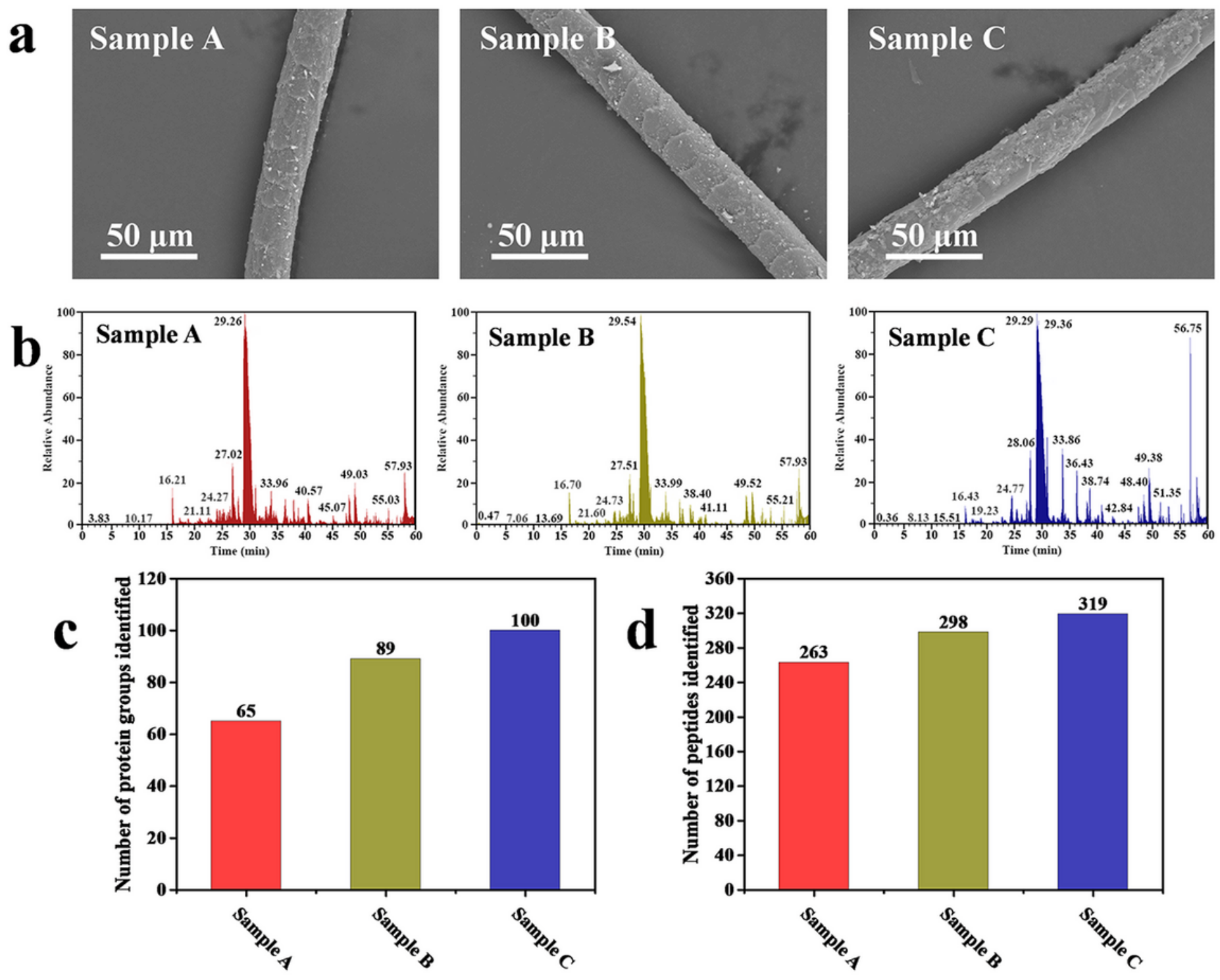

\section{Figure 4}

Morphologies and LC-MS/MS results of ancient hair textiles. (a) SEM images of the longitudinal surfaces for ancient hair textiles. (b) Base peak chromatograms of ancient hair textiles. Protein group number (c) and peptide number (d) of ancient hair textiles (Sample A (Xiaohe cemetery), Sample B (Shanpula cemetery), Sample $C$ (Shanpula cemetery)) identified by comparison against the mammalian keratins Uniprot database. 


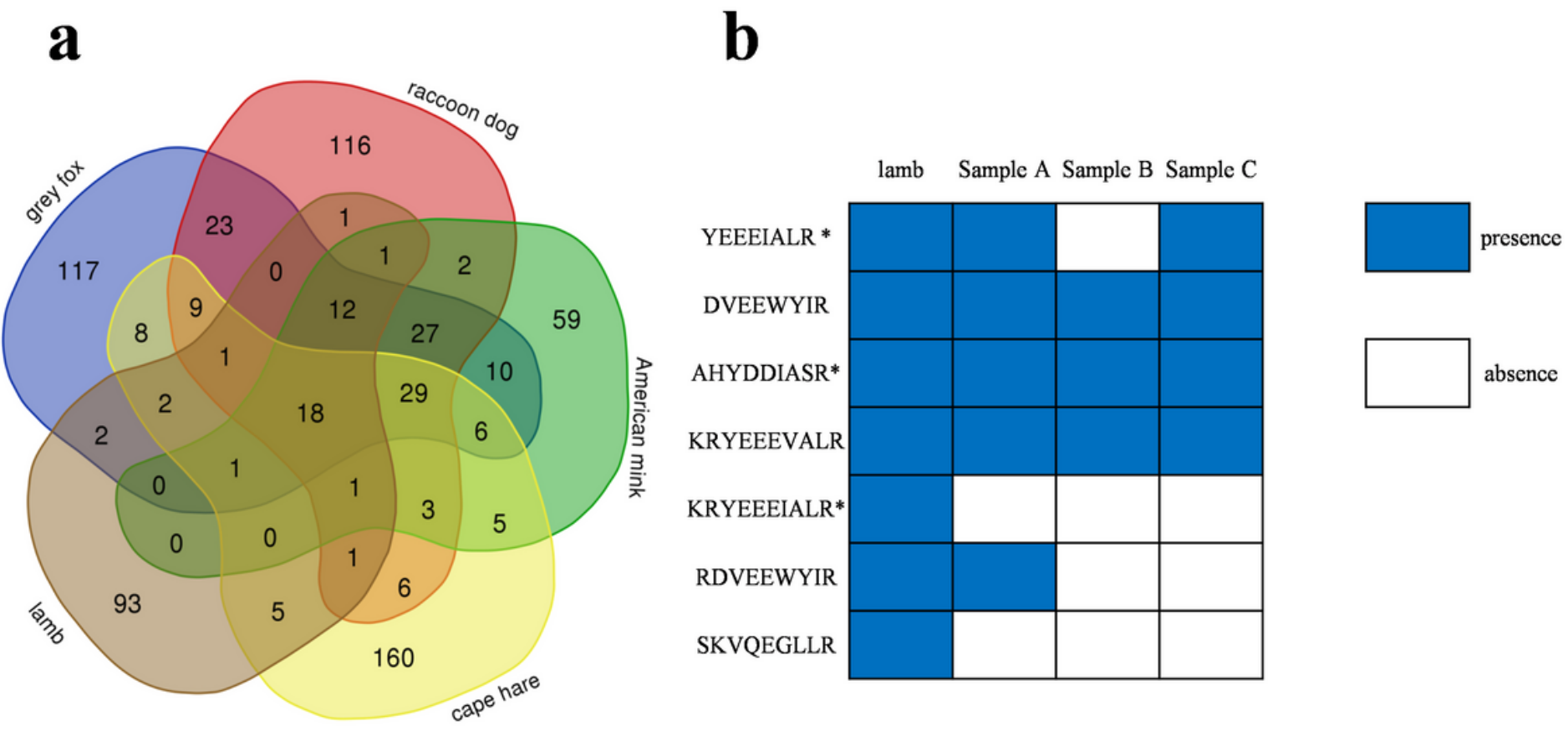

Figure 5

Species verification of ancient hair textiles through species-diagnostic peptides. (a) The overlap of peptides in different species identified by compar-ison against the corresponding species Uniprot databases. (b) Species-diagnostic peptides of sheep detected in the ancient hair textiles. (* peptide exists in protein biomarker E3VW87)

\section{Supplementary Files}

This is a list of supplementary files associated with this preprint. Click to download.

- Supportinglnformation.doc 